Bull. Egypt. Soc. Physiol. Sci. Vol. (41) Issue (1), 68-83

\author{
Bull. of Egyp. Soc. Physiol. Sci. \\ (Official Journal of Egyptian Society for Physiological Sciences) \\ (pISSN: 1110-0842; eISSN: 2356-9514)
}

\title{
Role of Adenosine Triphosphate Sensitive Potassium Channels in Glucocorticoid Induced Myopathy of a Rat Model
}

\section{Asmaa Yehia Moustafa Hatem, Mohamed EL Sayed EL Refaay Ghalwash, Abeer Foad Mostafa, Mohammed Elmahdy EI Hosiny Sarhan.}

Department of Medical Physiology, Faculty of Medicine, Mansoura University, Egypt.

Submit Date: April 27, 2020

Accept Date: May 17, 2020

Available Online : Jan 1, 2021

\section{Keywords}

- $\quad$ Glucocorticoids

- Mmyopathy

- $\quad$ kATP channels

- $\quad$ Insulin like growth factor (IGF-1)

\begin{abstract}
Objective: To clarify adenosine triphosphate sensitive potassium (KATP ) channels' role in glucocorticoid 1induced myopathy induction. Materials and methods: 48 adult male rats divided into two experiments. Experiment I: 32 rats divided equally into four group; Group I: control group, Group II: (Dexa group) (Dexamethasone $500 \mu \mathrm{g} / \mathrm{kg}$ once daily i.p), Group III (Dexa+ Nicorandil) (Nicorandil (3 $\mathrm{mg} / \mathrm{kg}$ ) per oral once daily), Group IV (Dexa+Glibenclamide) (Glibenclamide $(10 \mathrm{mg} / \mathrm{kg})$ per oral once daily) all for 10 days. By the end of the experiment, blood samples were collected for blood glucose, serum insulin, potassium and creatine kinaseMM. Tibialis anterior muscles were isolated for contractility recording, oxidative stress measurement, histopathological examination and immunohistochemistry for insulin like growth factor-1(IGF-1). Experiment II:16 rats (8 normal and 8 glucocorticoid induced myopathy rats). From each rat, the tibialis anterior was isolated and incubated with nicorandil $(100 \mu \mathrm{mol} / \mathrm{L}$ for 10 minutes) and glibenclamide $(1 \mu \mathrm{mol} / \mathrm{L}$ for 5 minutes) for contractility recording. Results: Contractile properties, histopathological changes and IGF-1 muscle expression are improved with glibenclamide (KATP channel blocker) and worsened with nicorandil (KATP channel opener). CK-MM level significantly increased with nicorandil, while normal with glibenclamide. Serum potassium and blood glucose significantly increased with nicorandil and decreased with glibenclamide. Serum insulin significantly decreased with nicorandil and increased with glibenclamide. The oxidative stress state insignificantly changed with both drugs ( $\mathrm{p}$ value $\leq 0.05$ was considered to be statistically significant). Conclusion: Opening of KATP channels seems to have a role in the induction of glucocorticoid-induced myopathy probably by decreasing IGF-1 expression.
\end{abstract}

Corresponding author: Asmaa Yehia, Tel: 0502943193 Physiology department, Faculty of medicine, Mansoura university, Egypt. E.mail asmaa_yehia@mans.edu.eg 


\section{INTRODUCTION}

In 1932, glucocorticoid-induced myopathy was first described in Cushing's syndrome (1). Patients with excess endogenous glucocorticoids develop severe proximal muscle wasting and weakness. About $60 \%$ of the patients with Cushing's syndrome suffer from muscle weakness. Hence the use of glucocorticoids in treating several medical conditions has been on the increase, exogenous glucocorticoids have been featured as the most common cause of drug-induced myopathy (2).

Patient with glucocorticoid-induced myopathy struggle with day to day activities such as rising from a chair and climbing stairs. Glucocorticoids mostly impair proximal musculature and type II muscle fibers (3). Moreover, the management of this condition is challenging due to the limited options of treatment, the indispensability of glucocorticoids in certain medical conditions and the persistence of myopathy even after the correction of the state of hypercortisolism as a long lasting adverse effect of glucocorticoids on skeletal muscles (4).

Glucocorticoid-induced myopathy seems to be the cumulative result of many factors. Glucocorticoids induce muscle protein catabolism via proteolytic systems and interfere with protein synthesis. Glucocorticoids decrease insulin like growth factor (IGF-1) production while increase that of myostatin, two growth factors controlling muscle mass (5). IGF-1 increases protein synthesis and myogenesis and decreases proteolysis and apoptosis while myostatin exerts an opposing action. This is conducted through certain signaling pathways. IGF-
1/PI3K/Akt/mTOR signaling pathway is known to play a pivotal role in activating protein synthesis(6).

Also, glucocorticoid-induced myopathy can be attributed to mitochondrial dysfunction and produces muscle weakness by lowering serum potassium. Moreover, glucocorticoids exert their wide range of function through genomic action by altering genes activity and through non-genomic action by activating secondary messengers signaling pathways and interfering with ion channels protein (7). Yet, the involvement of ion channels infliction in glucocorticoid induced myopathy is not thoroughly investigated.

ATP sensitive potassium $\left(\mathrm{K}_{\text {ATP }}\right)$ channels belong to the inward rectifying potassium (Kir) channel family and are considered as metabolic sensors. $\mathrm{K}_{\mathrm{ATP}}$ channels are so named because they open as cellular ATP levels fall. They link cellular metabolism to membrane excitability. They are widely distributed in tissues including neurons, pancreatic beta cells, smooth, cardiac and skeletal muscles. The multi-level regulation by membrane phospholipids (PIP2), fatty acids (LC-Acyl-CoA), protein kinases (PKA, PKC), glycolytic enzymes, $\mathrm{pH}$, hypoxia and intracellular nucleotides ensures complexity of metabolic sensing by the $\mathrm{K}_{\mathrm{ATP}}$ channels. (8). Moreover, $\mathrm{K}_{\mathrm{ATP}}$ channels can be regulated by a wide range of Pharmacological agents which include potassium channel openers (KCOs) and channel blockers. The most commonly used class of $\mathrm{K}_{\mathrm{ATP}}$ channel blockers is the hypoglycemic sulfonylureas. The first generation of sulfonylureas has a relatively low affinity for channel blocking and includes drugs such as tolbutamide and chlorpropamide. The second generation has a higher affinity and 
includes agents such as glibenclamide, glipizide, and gliclazide (9). KCOs of widely different structures can cause $\mathrm{K}_{\text {ATP }}$ channels activation which leads to membrane hyperpolarization and reduced electrical activity. They include nicorandil, minoxidil, pinacidil, cromakalim, and diazoxide which are classified into several distinct groups (10)

In skeletal muscles, $\mathrm{K}_{\mathrm{ATP}}$ channels have a role in muscle fatigue as they are activated in response to reduction of $\mathrm{ATP} / \mathrm{ADP}$ ratio resulting in decline in action potential duration and hence force production which act as an outlet for the fatiguing muscle till recovery is achieved (11). Emerging evidences suggest that in skeletal muscles, $\mathrm{K}_{\text {ATP }}$ channels are coupled to the IGF1-PI3K-AktmTOR signaling pathway (12) It seems that glucocorticoids and $\mathrm{K}_{\mathrm{ATP}}$ channels share the same signaling pathways. So, the present study was designed to clarify the role of $\mathrm{K}_{\text {ATP }}$ channels in the induction of glucocorticoid induced myopathy.

\section{Materials and Methods}

\section{Chemicals}

Dexamethasone sodium phosphate ampoules, Glibenclamide, Nicorandil were procured from Amriya, Sanofi and Merck Ltd for pharmaceutical industries, Egypt respectively.

\section{Animals}

Forty-eight adult male Sprague Dawely rats weighing between 170-230 g, aging 4-6 months were enrolled in this study. Animals were bred and housed in the animal house of the Medical Experimental Research center (MERC), Mansoura University, at controlled environmental conditions (temperature of $24{ }^{\circ} \mathrm{C}$ and 12 hours light/dark cycles) fed a standard laboratory chow and had a free access to tap water. All experimental protocols were approved by our local committee of animal care and ethics. (Code\# MS.18.12.390)

\section{Induction of glucocorticoid induced myopathy rat model}

For induction of glucocorticoid induced myopathy, rats were injected with Dexamethasone $(500 \mu \mathrm{g} / \mathrm{kg}$ body mass, intra-peritoneal) once a day for 10 day (13).

\section{Experimental Groups}

Rats were divided into two experiments. Experiment I included 32 rats divided into four groups, eight rats per each. The rats of group I served as normal control group. Group II is myopathic group (Dexa group) whose rats received dexamethasone $500 \mu \mathrm{g} / \mathrm{kg}$ once daily i.p. for 10 days. Group III (Dexa+ Nicorandil) rats were treated with dexamethasone $500 \mu \mathrm{g} / \mathrm{kg}$ once daily i.p. + nicorandil (3 mg/kg) per oral once daily for 10 days. Group IV (Dexa+ Glibenclamide) rats received dexamethasone $500 \mu \mathrm{g} / \mathrm{kg}$ once daily i.p. + glibenclamide $(10 \mathrm{mg} / \mathrm{kg})$ per oral once daily for 10 days. Experiment $\mathrm{II}$ included 16 rats (8 normal rats and 8 glucocorticoid induced myopathy rats). From each rat, the tibialis anterior muscle was isolated and incubated with Nicorandil and glibenclamide for contractility recording.

Blood samples collection and obtaining samples of tibialis anterior muscles

Rats were anesthetized with $10 \mathrm{ml}$ halothane and sacrificed with cervical dislocation, the anterior chest wall was opened and blood sample was collected by cardiac puncture (14). Blood was centrifuged at 2000 rotation per minute (rpm) for 
10 minutes to obtain serum. Then sera were frozen and stored at $-20{ }^{\circ} \mathrm{C}$ for biochemical tests.

After blood samples collection, hind limbs were separated and tibialis anterior muscles were dissected. Then, the right tibialis anterior was used for contractility recordings and left in formalin $(10 \%)$ for further histopathological and immunostaining tests. The left muscle was taken for measurement of stress markers using liquid nitrogen for preservation.

\section{Contractility recording}

Contractility recording was achieved by using Biopac ${ }^{\circledR}$ system inc (BSL 3.7.5 software), (MP36) data analysis unit, BIOPAC force transwicer assembly (SS12LA), BIOPAC BSLSTM stimulator and needle electrodes (ELSTM2). The following parameters were recorded after sacrifice and dissection of tibialis anterior muscle: (maximal twitch force, time to peak, half-relaxation time, maximum tetanic force, force after tetanic contraction). Fatigue induction by repetitive tetani until the force declined to about $50 \%$ of the original force or until $10 \mathrm{~min}$ passed, whichever occurred first and calculating time to fatigue (15).

\section{i. Biochemical Measurements}

\section{Serum Creatine kinase-MM (CK-MM) assay:}

Measured using commercially available kits according to the manufacturer's instructions. Using CK-MM sensitive ELISA kit purchased from abnova (Taiwan) Cat\#: KA2072

Blood glucose, Serum insulin and potassium level:

Measured by using commercially available kits according to the manufacturer's instructions. glucose and insulin were measured by kits for rat (ELISA) obtained from company of Sun-Red biology and technology, Shanghai cat\#: 201-11-
0708. Serum potassium was measured by Potassium (K) Colorimetric Assay Kit purchased from Elabscience ${ }^{\circledR}$ (USA) Cat\# E-BC-K279-M

Assessment of oxidative stress markers in muscle tissue:

A small part of the tibialis anterior muscle was taken, homogenized in $0.02 \mathrm{M}$ sodium phosphate buffer, pH 7.4 (1:4 water/volume) and aliquoted into different ependorffs. Reduced glutathione (GSH) (antioxidant) and malondialdehyde (MDA) (lipid peroxidation marker) were measured by colorimetric kits (Bio-Diagnostics, Giza, Egypt) according to the manufacturer's instructions. (Cat\# GR 25 11) (cat\# MD 25 29) respectively.

\section{Histopathological examination}

Washing of collected muscle tissues with saline and then fixation in $10 \%$ buffered neutral formalin solution were done immediately. After fixation, the muscle tissue was processed embedding in paraffin. After that, the muscle tissue was sectioned and stained with hematoxylin and eosin (H\&E). Histopathological evaluation was performed under light microscope. Characteristics of glucocorticoid induced myopathy are hyaline degenerative changes within the muscle fibers associated with fibrosis, atrophic changes and scanty vacuoles (16).

\section{Immunohistochemical examination for insulin} like growth factor 1 (IGF-1):

The tissue sections were deparaffinized, rehydrated, washed, immersed in $3 \% \mathrm{H} 2 \mathrm{O} 2$, and then digested with pepsin for antigen retrieval. After the blocking of unspecific binding by serum, the sections were incubated with rabbit polyclonal anti-rat IGF-1 antibodies (1:100, A11985, Abclonal, USA) at $40^{\circ} \mathrm{C}$ overnight. Diaminobenzidine / peroxidase substrate was used to produce a brown-colored signal. The section 
was counterstained, dehydrated, cleared, and cover slipped. Phosphate buffered solution (PBS) was used to replace primary antibody and adjacent sections will be used as negative control. insulin like growth factor-1 was quantified as the percent of muscle area occupied by positive staining (calculated by averaging the values from ten fields at $10 \times$ magnification) for each muscle area by using image $\mathrm{J}$ software.

\section{Computer Assisted digital image analysis (Digital morphometric study)}

Slides were photographed using Olympus ${ }^{\circledR}$ digital camera installed on Olympus ${ }^{\circledR}$ microscope with $1 / 2 \mathrm{X}$ photo adaptor, using $40 \mathrm{X}$ objective. The result images were analyzed on Intel ${ }^{\circledR}$ Core $15 \AA$ based computer using VideoTest ${ }^{\circledR}$ Morphology ${ }^{\circledR}$ software (Russia) with a specific built-in routine for $\%$ area measurement. 5 slides from each case were prepared, 5 random fields from each slide were analyzed.

\section{Statistical analysis}

Data were entered and statistically analyzed using the Statistical Package for Social Sciences (SPSS) version 16. The data were expressed as Mean \pm SD. Comparison for parametric data were carried out by analysis of variance (ANOVA) followed by Tukey's post hoc analysis for intergroup multiple comparisons. $\mathrm{P}<0.05$ was considered significant.

\section{Results}

\section{Contractility recording}

Regarding the isometric contractile properties in experiment I, Figure (1) and table (1) show a significant decrease in forces of contraction (maximum twitch force, maximum Tetanic force, force after tetanic contraction) and resistance to fatigue together with a significant increase in twitch times (time to peak twitch, half relaxation time twitch) in the dexa group when compared to the control group. These parameters were significantly enhanced in glibenclamide treated group and worsened in nicorandil treated group when compared to the dexa group.

In experiment II, figure (2) and table (2) show the results of Contractility recording where the control+ nicorandil group showed a significant decrease in forces of contraction (maximum twitch force, maximum Tetanic force) and resistance to fatigue, while it showed a significant increase in contraction times (time to peak twitch, half relaxation time twitch) when compared to the control group. However, it showed a significant increase in force of recovery. Also, the control+ glibenclamide group showed a normal contractile times and forces of contraction, yet a decreased force of recovery when compared to the control group. Both dexa+ nicorandil and dexa+ glibenclamide groups show almost the same changes as the same groups in experiment I .

Table (1): Contractility parameters in different groups in experiment (I)

\begin{tabular}{|l|c|c|c|c|}
\hline & control & Dexa & Dexa+Nico & Dexa+Gliben \\
\hline Max. twitch force (g.) & $13.3 \pm 0.6$ & $4.9 \pm 0.3^{*}$ & $2.4 \pm 0.3^{* \#}$ & $8.7 \pm 0.3^{* \# \$}$ \\
\hline Time to peak (sec.) & $0.08 \pm 0.005$ & $0.9 \pm 0.05^{*}$ & $1.8 \pm 0.1^{* \#}$ & $0.1 \pm 0.01^{* \# \$}$ \\
\hline Half relax time (sec.) & $0.2 \pm 0.01$ & $1.2 \pm 0.04^{*}$ & $1.9 \pm 0.04^{* \#}$ & $0.5 \pm 0.06^{* \# \$}$ \\
\hline Max. tetanic force (g.) & $23.3 \pm 0.5$ & $8.6 \pm 0.4^{*}$ & $4.7 \pm 0.4^{* \#}$ & $16.6 \pm 0.5^{* \# \$}$ \\
\hline Time to fatigue (sec.) & $95.4 \pm 1.7$ & $32.9 \pm 1.5^{*}$ & $23.3 \pm 0.8^{* \#}$ & $57.9 \pm 1^{* \# \$}$ \\
\hline $\begin{array}{l}\text { Force after tetanic } \\
\text { contraction (g.) }\end{array}$ & $6.2 \pm 0.4$ & $1.6 \pm 0.1^{*}$ & $0.6 \pm 0.1^{* \#}$ & $3.7 \pm 0.4^{* \# \$}$ \\
\hline
\end{tabular}

Test used: One way ANOVA followed by post-hoc tukey. Values are expressed as means \pm SD.Dexa= dexamethasone injected group. Gliben= glibenclamide KATP channel blocker. Nico= nicorandil KATP channel opener. *: Significant vs control group. \#: Significant vs Dexa group \$: Significant vs Dexa + nico group. p value $\leq 0.05$ was considered to be statistically significant. 
Table (2): Contractility parameters in different groups in experiment (II)

\begin{tabular}{|l|c|c|c|c|c|l|}
\hline & control & $\begin{array}{c}\text { Control+ } \\
\text { Gliben }\end{array}$ & $\begin{array}{c}\text { Control }+ \\
\text { Nico }\end{array}$ & Dexa & $\begin{array}{c}\text { Dexa }+ \\
\text { Gliben }\end{array}$ & \multicolumn{1}{|c|}{$\begin{array}{c}\text { Dexa }{ }^{+} \\
\text {Nico }\end{array}$} \\
\hline $\begin{array}{l}\text { Max. Twitch force } \\
\text { (g.) }\end{array}$ & $13.2 \pm 0.5$ & $13 \pm 0.4$ & $9.7 \pm 0.2^{\mathrm{ab}}$ & $5 \pm 0.3^{\mathrm{abc}}$ & $7.9 \pm 0.3^{\mathrm{abcd}}$ & $2.5 \pm 0.3^{\mathrm{abcde}}$ \\
\hline Time to peak (sec.) & $0.08 \pm 0.009$ & $0.08 \pm 0.007$ & $0.1 \pm 0.02^{\mathrm{ab}}$ & $0.9 \pm 0.04^{\mathrm{abc}}$ & $0.1 \pm 0.02^{\mathrm{abd}}$ & $1.8 \pm 0.1^{\mathrm{abcde}}$ \\
\hline $\begin{array}{l}\text { Half relaxation time } \\
\text { (sec.) }\end{array}$ & $0.2 \pm 0.009$ & $0.2 \pm 0.007$ & $0.4 \pm 0.06^{\mathrm{ab}}$ & $1.2 \pm 0.06^{\mathrm{abc}}$ & $0.5 \pm 0.06^{\mathrm{abcd}}$ & $2 \pm 0.1^{\mathrm{abcde}}$ \\
\hline $\begin{array}{l}\text { Max. Tetanic force } \\
\text { (g.) }\end{array}$ & $23 \pm 0.4$ & $22.9 \pm 0.5$ & $18.1 \pm 0.3^{\mathrm{ab}}$ & $8.7 \pm 0.4^{\mathrm{abc}}$ & $16.3 \pm 0.3^{\mathrm{abcd}}$ & $4.9 \pm 0.2^{\mathrm{abcde}}$ \\
\hline Time to fatigue (sec.) & $97.1 \pm 0.4$ & $96.2 \pm 0.7$ & $71.8 \pm 1.01^{\mathrm{ab}}$ & $32 \pm 1.6^{\mathrm{abc}}$ & $54.6 \pm 1.4^{\mathrm{abcd}}$ & $24.5 \pm 0.9^{\mathrm{abcde}}$ \\
\hline $\begin{array}{l}\text { Force after tetanic } \\
\text { contraction (g.) }\end{array}$ & $6.1 \pm 0.3$ & $3.8 \pm 0.5^{\mathrm{a}}$ & $6.3 \pm 0.3^{\mathrm{b}}$ & $1.7 \pm 0.1^{\mathrm{abc}}$ & $4.1 \pm 0.2^{\mathrm{abcd}}$ & $0.6 \pm 0.04^{\mathrm{abcde}}$ \\
\hline
\end{tabular}

'Test used: One way ANOVA followed by post-hoc tukey. Values are expressed as means \pm SD. Dexa= dexamethasone Gliben $=$ glibenclamide. $\mathrm{Nico}=$ nicorandil . a: significant vs control. b: significant vs Control+ Gliben. c: significant vs Control+ Nico. d: significant vs Dexa. e: significant vs Dexa+ gliben. $p$ value $\leq 0.05$ was considered to be statistically significant.
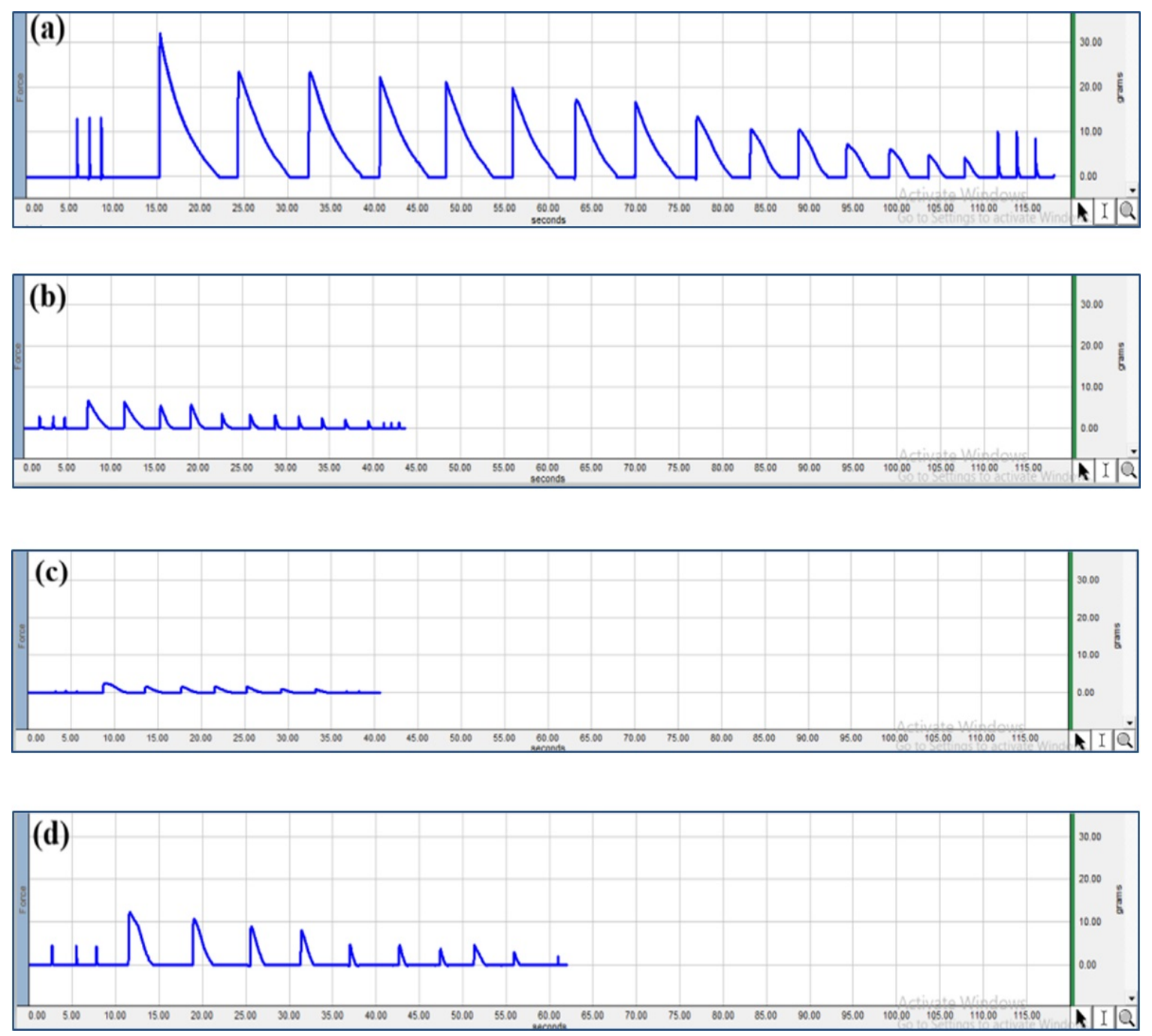

Figure (1): Traces of contractile activities of tibialis anterior in experiment I. (a) Control group.(b) Dexa group (c) Dexa+ Nico group (d) Dexa+ Gliben group. 

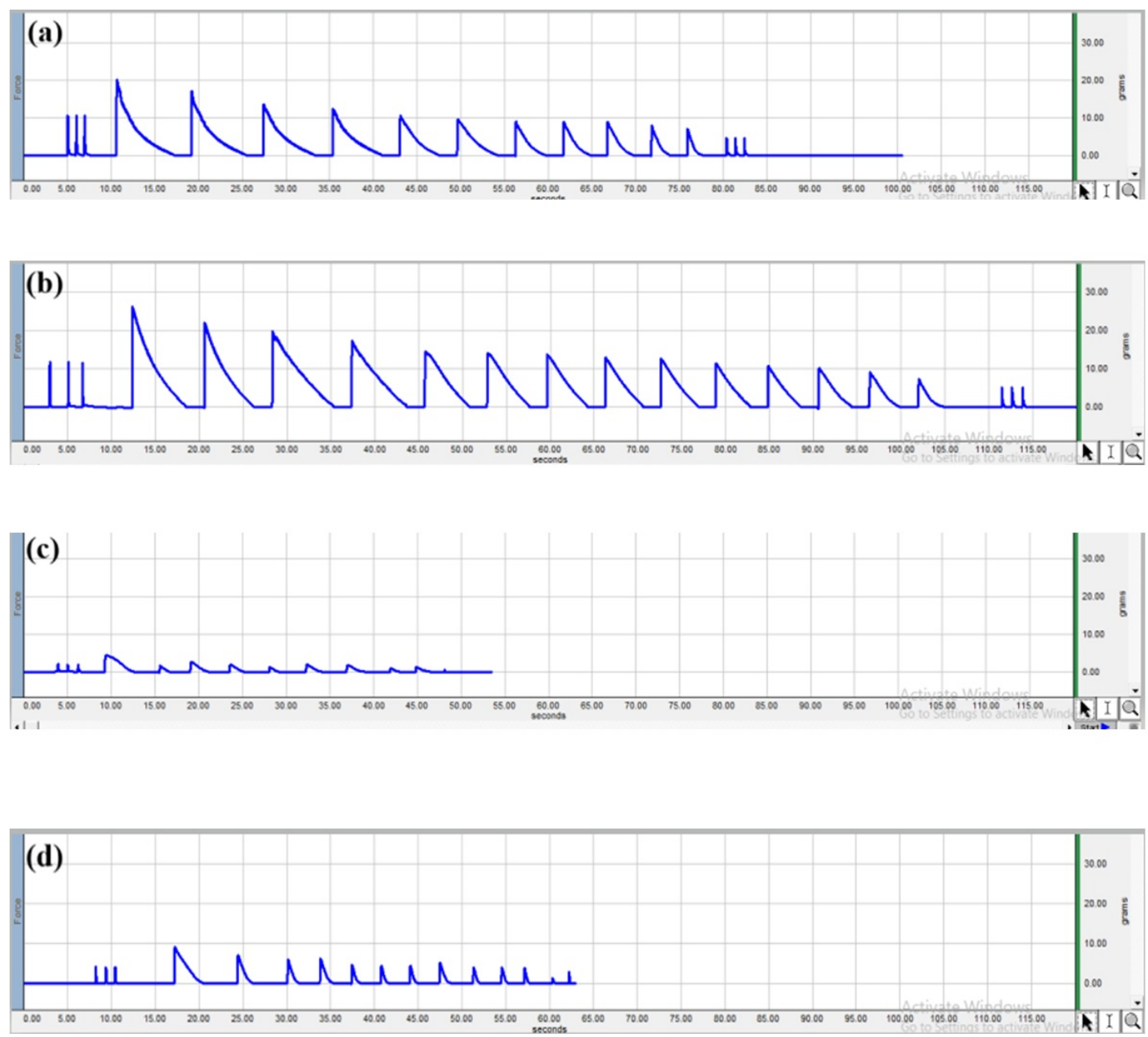

Figure (2): Traces of contractile activities of tibialis anterior in experiment II. (a) Control + Nico group. (b) Control + Glibenclamide. (c) Dexa + Nico. (d) Dexa + Glibenclamide group.

\section{Biochemical analysis}

As regard the biochemical analysis, table (3) show a significant increase in blood glucose level in the dexa group compared to the control group. This hyperglycemic state is improved with glibenclamide and worsened significantly with nicorandil when compared to the dexa group. Moreover, serum insulin was significantly reduced in dexa group with more reduction demonstrated in nicorandil treated rats. Also, serum potassium levels were significantly decreased in all groups compared to the control group. However, serum potassium was significantly increased in nicorandil group and reduced in glibenclamide group when compared to the dexa group. As for the serum CK-
MM, all groups showed a normal level except for the nicorandil treated group which showed a significant increase.

\section{Oxidative stress markers in muscle tissue}

Table (4) shows a worsened oxidative stress condition in the tibialis anterior muscle of dexamethasone treated rats which is demonstrated in significantly elevated MDA level and reduced GSH level compared to the control group. However, both glibenclamide and nicorandil treated rats showed no change in the oxidative state from the dexa group.

\section{Histopathological examination}

Figure (3) shows normal architecture of muscle fibers with regular parallel arrangement and 
peripherally placed nuclei in the tibialis anterior specimen obtained from the control group. Dexa treated muscles showed hyaline degenerative changes with atrophic muscle fibers associated with extensive fibrosis. Nicorandil treated muscles
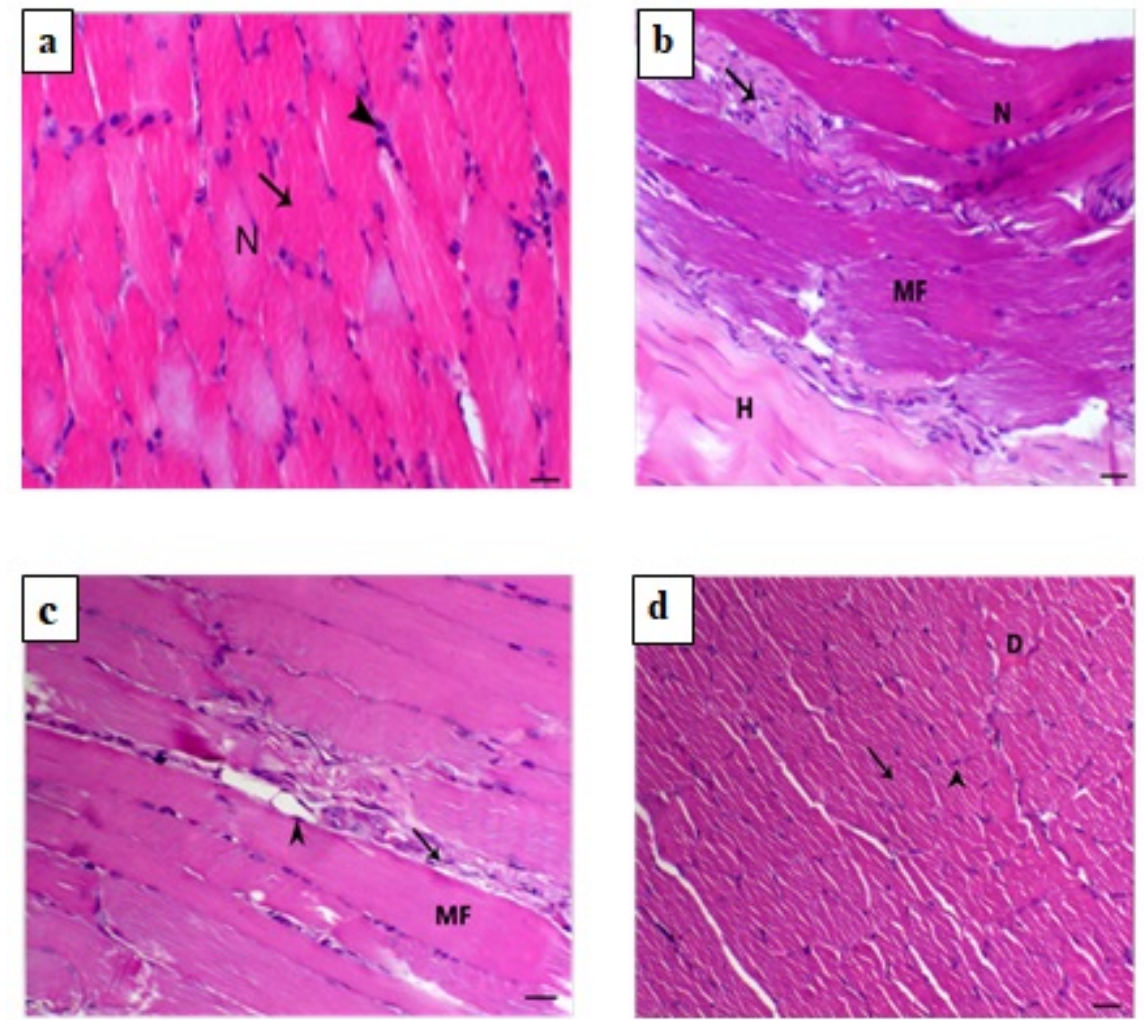

Figure (3): A) Control group. Muscle specimen showing normal architecture of muscle fibers with regular parallel arrangement (arrow), hexagonal contour and peripherally placed nuclei $(\mathrm{N})$. The sarcoplasm appears brightly eosinophilic with basophilic granules more toward the periphery of the muscle fiber (arrowhead) (B): Dexa group. Muscle specimen showing atrophic muscle fibers (MF) with hyaline degenerative changes $(\mathrm{H})$ within the muscle fibers associated with fibrosis (arrow) and aggregated nuclei (N) C) Dexa + Nico group. Muscle specimen showing atrophic muscle fibers (MF) with focal interstitial fibrosis (arrow), aggregated nuclei $(\mathrm{N})$ and scanty vacuoles (arrowhead) D) Dexa + Gliben group. Muscle specimen showing showing normal muscle fibers with minimal atrophic changes (arrow) with peripherally located nuclei (arrowhead) and minimal degenarative changes (D) (H\&E, 400x).

Table (3): Results of blood glucose, serum insulin, potassium and CK-MM in different groups.

\begin{tabular}{|l|c|c|c|c|}
\hline & control & Dexa & Dexa+Nico & Dexa+Gliben \\
\hline $\begin{array}{l}\text { Blood glucose level } \\
\text { (mg/dl) }\end{array}$ & $93.1 \pm 6.9$ & $228.5 \pm 17.8^{*}$ & $300.1 \pm 14.4^{* \#}$ & $137.7 \pm 13.4^{* \# \$}$ \\
\hline $\begin{array}{l}\text { Serum insulin level } \\
\text { (IU/ml) }\end{array}$ & $11.3 \pm 0.2$ & $9.7 \pm 0.3^{*}$ & $8.2 \pm 0.2^{* \#}$ & $11.1 \pm 0.4^{\# \$}$ \\
\hline $\begin{array}{l}\text { Serum potassim level } \\
\text { (mEq/L) }\end{array}$ & $14.1 \pm 0.4$ & $9.8 \pm 0.8^{*}$ & $11.9 \pm 0.2^{* \#}$ & $9.2 \pm 0.5^{* \# \$}$ \\
\hline $\begin{array}{l}\text { serum creatine kinase } \\
\text { (ng/ml) }\end{array}$ & $4.8 \pm 0.2$ & $5.01 \pm 0.5$ & $5.6 \pm 0.5^{* \#}$ & $5 \pm 0.4^{\$}$ \\
\hline
\end{tabular}

Test used: One way ANOVA followed by post-hoc tukey. Values are expressed as means \pm SD. Dexa= dexamethasone injected group. Gliben= glibenclamide $\mathrm{K}_{\mathrm{ATP}}$ channel blocker. Nico= nicorandil $\mathrm{K}_{\mathrm{ATP}}$ channel opener. *: Significant vs control group. \#: Significant vs Dexa group \$: Significant vs Dexa + nico group. p value $\leq 0.05$ was considered to be statistically significant. 
Table (4): Results of Oxidative stress markers in different groups

\begin{tabular}{|l|c|c|c|c|}
\hline & control & Dexa & Dexa+Nico & Dexa+Gliben \\
\hline $\begin{array}{l}\text { MDA (nmol/0.08g. } \\
\text { tissue) }\end{array}$ & $246.7 \pm 6$ & $572.9 \pm 15.8^{*}$ & $565.4 \pm 16.8^{*}$ & $577.4 \pm 12.6^{*}$ \\
\hline $\begin{array}{l}\text { GSH (mmol/0.08g. } \\
\text { tissue) }\end{array}$ & $33.9 \pm 1.4$ & $14.7 \pm 0.6^{*}$ & $15.1 \pm 0.9^{*}$ & $14.3 \pm 1.1^{*}$ \\
\hline
\end{tabular}

Test used: One way ANOVA followed by post-hoc tukey. Values are expressed as means \pm SD.

Dexa $=$ dexamethasone injected group. Gliben $=$ glibenclamide KATP channel blocker. Nico= nicorandil KATP channel opener. *: Significant vs control group. $\mathrm{p}$ value $\leq 0.05$ was considered to be statistically significant.

\section{Immunohistochemical examination for insulin}

like growth factor 1 (IGF-1)

Table (5) and figures (4,5) show a decreased immunopositivity for IGF-1 with few positive muscle fibers in muscles obtained from the dexa group. However, muscle specimens obtained from the Dexa+Nico group showed a more decreased immunopositivity for IGF-1 with scattered positive muscle fibers. Moreover, muscle specimens obtained from the Dexa+ Gliben group showed increased IGF-1 positive muscle fibers that are evenly distributed and oriented in the longtudinal axis of the muscle fibers.

Table (5): Density of IGF-1 in muscles in different groups

\begin{tabular}{|l|c|c|c|c|}
\hline & control & Dexa & Dexa+Nico & Dexa+Gliben \\
\hline Density of IGF-1 (\%) & $2.9 \pm 0.02$ & $1.2 \pm 0.01^{*}$ & $0.9 \pm 0.01^{* \#}$ & $2.2 \pm 0.009^{* \# \$}$ \\
\hline
\end{tabular}

Test used: One way ANOVA followed by post-hoc tukey. Values are expressed as means \pm SD.

Dexa $=$ dexamethasone injected group. Gliben= glibenclamide KATP channel blocker. Nico= nicorandil KATP channel opener. *: Significant vs control group. \#: Significant vs Dexa group \$: Significant vs Dexa + nico group. $p$ value $\leq 0.05$ was considered to be statistically significant.
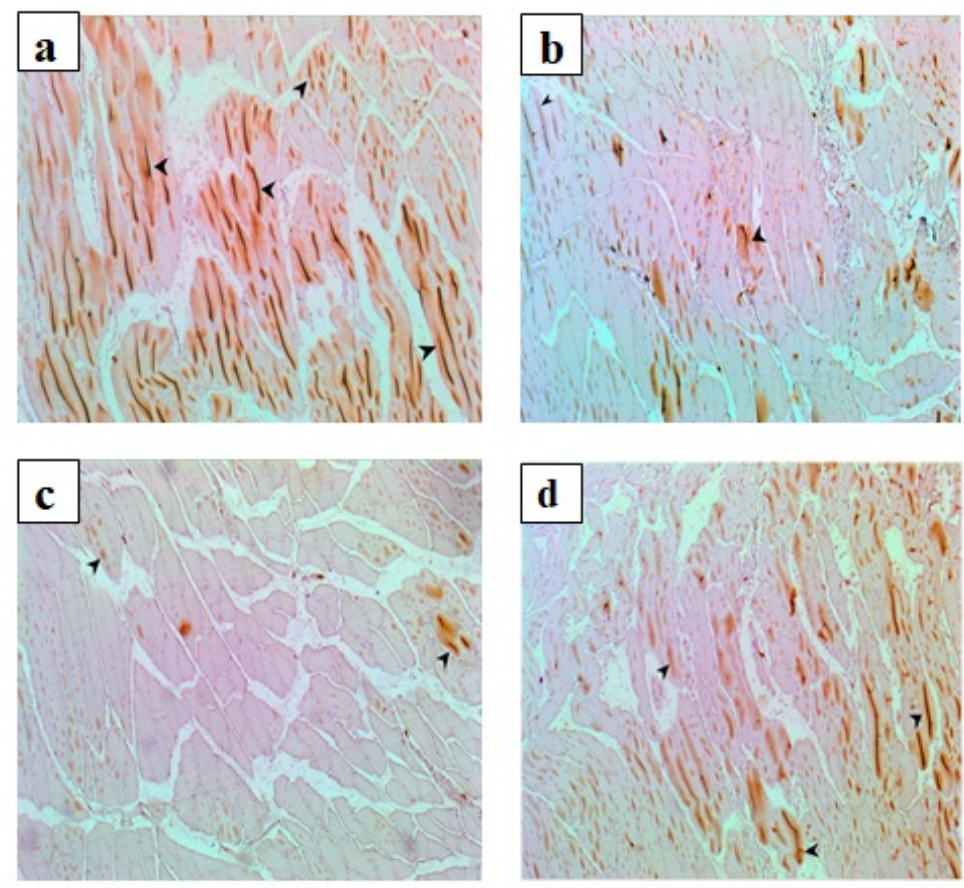

Figure (4): A) Control group. Muscle specimen showing immunopositivity for IGF-1 in rat muscle. IGF-1 is evenly distributed and oriented in the longitudinal axis of muscle fibers. B) Dexa group. Muscle specimen showing decreased immunopositivity for IGF-1 with scattered IGF-1 positive fibers . C) Dexa + Nico group. Muscle specimen showing marked decrease in immunopositivity for IGF-1 positive fibers D) Dexa + Gliben group. Muscle specimen showing enhanced immunopositivity for IGF-1 positive fibers (DAB, 400x). 


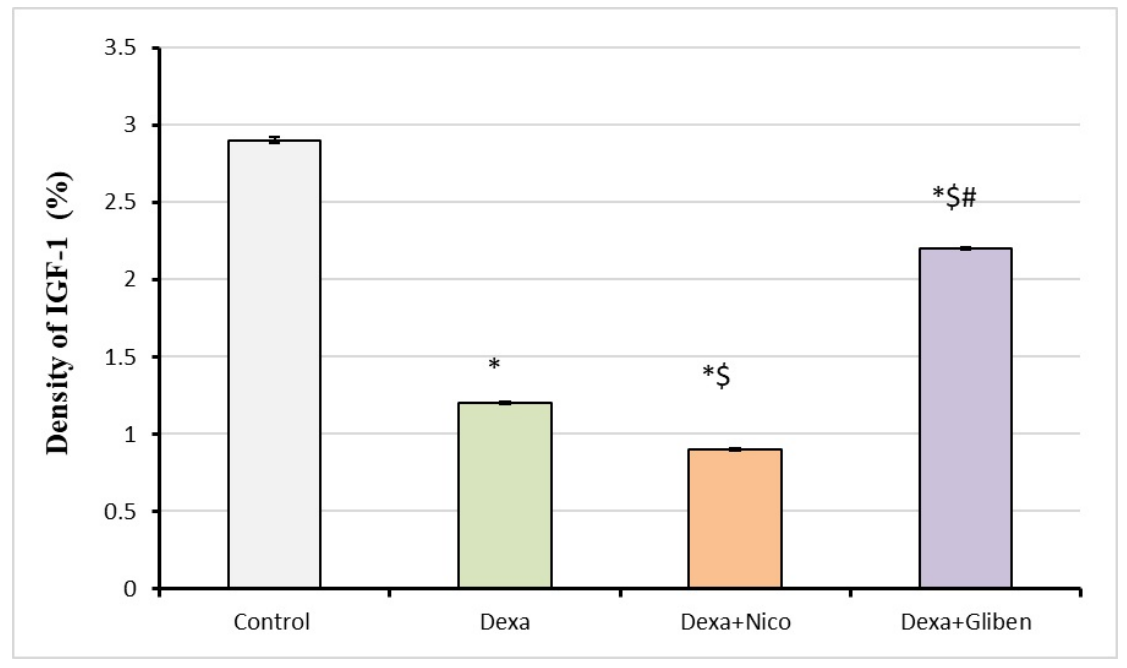

Figure (5): Density of insulin like growth factor-1 in different groups. * means significant vs control group, $\$$ means significant vs Dexa group and \# means significant vs Dexa+Nico group.

\section{Discussion}

Glucocorticoid-induced myopathy is acknowledged as one of the detrimental outcomes of hypercortisolism as in Cushing's syndrome and chronic treatment with high doses of glucocorticoids. Glucocorticoids set off myopathy through a multifactorial mechanism: mitochondrial dysfunction, hypokalemia and most predominantly through decreasing insulin-like growth factor (IGF-1) production, a skeletal muscle protein synthesis promoting growth factor and activating proteolytic systems via inhibiting IGF1/PI3K/Akt/mTOR signaling pathway ending up in muscle atrophy and verifying the reputation of glucocorticoids as metabolic stressors.

According to Huang et al., we used a ten days dexamethasone injection model $(500 \mu \mathrm{g} / \mathrm{kg}$ body mass) to induce skeletal muscle atrophy in rats (17). The induction of myopathy was confirmed by histopathological changes: hyaline degenerative changes within the muscle fibers associated with fibrosis, atrophic changes and scanty vacuoles which is consistent with previous studies $(1,16$, 18, 19). Dexamethasone was chosen based on the reported higher incidence of glucocorticoidinduced myopathy with fluorinated steroids such as dexamethasone more than with non-fluorinated types (20). Tibialis anterior muscle was chosen for the study of the injurious effect of glucocorticoids on skeletal muscles due to the higher incidence of glucocorticoid-induced myopathy in type п muscle fibers represented in tibialis anterior muscle. They possess high amounts of glucocorticoid receptors besides more numerous $\mathrm{K}_{\text {ATP }}$ channels $(3,21)$.

$\mathrm{K}_{\text {ATP }}$ channels are metabolic sensors that operate as an outlet for skeletal muscles during fatigue and debilitating stressors. During fatigue, $\mathrm{K}_{\mathrm{ATP}}$ channels allow potassium efflux, hyperpolarizing the cell membrane, slowing down the muscle action and preserving energy till relief is achieved. $\mathrm{K}_{\mathrm{ATP}}$ channels not only affect the cell excitability and link it to the cell metabolic condition but also interact with intracellular signaling pathways, cell enzymes, and transcriptional factors (8). It is not possible but to notice the mischievous metabolic effects of glucocorticoids and how this could allow a link between them and $\mathrm{K}_{\mathrm{ATP}}$ channels. The role of $\mathrm{K}_{\mathrm{ATP}}$ channels is elucidated by blocking and 
opening them using glibenclamide and nicorandil respectively. Glibenclamide was chosen as a channel blocker as it belongs to the second generation of sulfonylureas which has a higher affinity for channel blocking and a better hypoglycemic effect (9). Also, glibenclamide was claimed to affect the muscle IGF-1 expression and muscle protein content (12). Nicorandil was chosen as a channel opener due to its hyperkalemic effect, its effect on skeletal muscle contractility, its claimed relation to IGF-1 expression, and the claim of being a myopathic drug (22-24).

In the present study, glibenclamide seems to mildly enhance the histopathological changes when compared to the myopathic group while nicorandil exhibits atrophic changes similar to the myopathic group. The effects of nicorandil at hand is harmonious to Chasioti et al., who listed nicorandil as a myopathy inducing drug (24). Yet, the effects of glibenclamide is paradoxical to Mele et al., who pointed out a potential atrophic effect of glibenclamide on skeletal muscles(25). The ameliorated histopathological findings in the glibenclamide-treated rats could be explained by the subsequently demonstrated results of IGF1expression and potassium level which imply a possible improvement in protein synthesis.

The decreased expression of IGF-1 in myopathic rats is consistent with the inhibitory effect of glucocorticoids on IGF-1 and its anabolic pathway as reported in previous studies $(20,26-28)$. IGF-1 expression is improved in glibenclamide-treated rats and worsened in the nicorandil-treated rats that is consistent with the histopathological findings and the contractility records in the present study. There has been conflicted reports regarding the expression of IGF-1 upon opening or closing the
$\mathrm{K}_{\text {ATP }}$ channels. Tricarico et al., stated that the IGF1-PI3K-Akt-mTOR signaling pathway is activated upon opening of the $\mathrm{K}_{\mathrm{ATP}}$ channels and the opposite upon closing, ending up in a decreased protein content with glibenclamide and an increased protein content with diazoxide, another $\mathrm{K}_{\mathrm{ATP}}$ channels opener (12). However, Haba et al., issued an antagonistic relation between IGF1-PI3K-Akt-mTOR pathway and $\mathrm{K}_{\text {ATP }}$ channels function, where inhibited channel function results in activation of this pathway (29). The presented results in this study is more coherent with the latter opinion.

This could be explained as follows. Glucocorticoids inhibit the IGF1-PI3K-Akt-mTOR pathway eliminating its anabolic effect. This inhibited cascade by glucocorticoids ends up activating AMP-activated protein kinase (AMPK) and fork head box O (FOXO) transcription factors. The activated AMPK stimulates the $\mathrm{K}_{\mathrm{ATP}}$ channel opening and expression. Also, FOXO transcription factors were found to enhance the $\mathrm{K}_{\mathrm{ATP}}$ channels expression, activity and sensitivity to metabolic stress (30-32). Moreover, the mischievous metabolic effects of glucocorticoids could function as stimulants for the $\mathrm{K}_{\mathrm{ATP}}$ channels. Glucocorticoids were proven to cause intracellular ATP deprivation, increased ADP concentration and increased fatty acids oxidation which are all stimulants for $\mathrm{K}_{\text {ATP }}$ channels activity $(11,33-35)$. Furthermore, sierra et al., stated that blocking or disruption of the $\mathrm{K}_{\mathrm{ATP}}$ channels would result in inactivation of the atrophic FOXO (36).

Non-elevated CK-MM levels in dexamethasonetreated rats could be explained that glucocorticoids don't destruct muscle fibers and hence allow CK to leak from cells into blood serum, but rather hold 
off protein synthesis (37). The glibenclamide group exhibited normal levels of CK-MM which imply a non-exacerbation of the myopathic condition. However, the increased CK-MM in nicorandil-treated group suggests a deteriorated muscle fibers state. The decreased expression of IGF-1 in nicorandil group is possibly accompanied by increased levels of AMPK. AMPK causes exclusion of $\mathrm{CK}$ from within the muscle fibers to minimize the decreased ATP level by CK (38). This could explain the increased level of CK-MM in nicorandil treated group.

Declined contractile properties in dexamethasone injected rats were reversed with glibenclamide treatment. This is coherent to Duty et al., who stated that the increased force upon $\mathrm{K}_{\mathrm{ATP}}$ channels blockage is attributable to decreased potassium efflux, shortening of action potential and increased calcium release and hence force of contraction (39). Contractile properties in nicorandil treated rats showed a decline in the force of contraction (maximum twitch and maximum tetanic force) and time to fatigue with a significant prolongation in time to peak and half relaxation times when compared to the control and the myopathic groups.

This could be explained by the reported hyperpolarization and decline in the action potential overshoot and duration upon the opening of the $\mathrm{K}_{\mathrm{ATP}}$ channels. The resulting decrease in membrane excitability and action potential amplitude leads to a decrease in calcium release and force of contraction (40-43). Yet, previous studies show an increase in the force of recovery after stopping of fatigue when using $\mathrm{K}_{\text {ATP }}$ channel opener (44) which is contradictory to the results in the current study that showed worsening in force of recovery in nicorandil-treated myopathic rats when compared to the control and Dexa groups. This could be explained by the subsequently demonstrated results of IGF-1expression and potassium level.__ The recorded contractile properties exhibit almost the same results in both experiments I and II. This implies that the displayed results of contractile properties are due to the involvement of skeletal $\mathrm{K}_{\mathrm{ATP}}$ channels not elsewhere $\mathrm{K}_{\mathrm{ATP}}$ channels. These diverse results in both nicorandil and glibenclamide-treated myopathic rats compared to the Dexa group imply a possible increased activity of $\mathrm{K}_{\mathrm{ATP}}$ channels in case of glucocorticoid-induced myopathy.

The continued deteriorated oxidative stress condition with both nicorandil and glibenclamide is not consistent with the previously demonstrated findings of both drugs on contractility, histopathological changes and IGF-1 expression in the present study. Yet, his might allude to exclusion of the $\mathrm{K}_{\mathrm{ATP}}$ channels from induction of oxidative stress in the case of glucocorticoid induced myopathy.

Glucocorticoids induce hypokalemia which was reported as a possible mechanism for muscle weakness while nicorandil induces extracellular increase of potassium after the already present hypokalemia with dexamethasone, depleting the muscle of its intracellular potassium which is needed for protein synthesis so, nicorandil could aid the anti-anabolic effect of glucocorticoids. Moreover, glibenclamide increases insulin which is proven to help intracellular potassium uptake (45, 46).

As regards serum insulin and glucose levels, nicorandil group exhibited lowered insulin levels and hyperglycemia which is countered in the glibenclamide group when compared to the 
myopathic group. This goes back to the effect of both drugs on the pancreatic $\mathrm{K}_{\mathrm{ATP}}$ channels. However, skeletal $\mathrm{K}_{\mathrm{ATP}}$ channels were suggested to have a role in the muscle glucose uptake which is disrupted upon opening of the $\mathrm{K}_{\text {ATP }}$ channel. Also, glucocorticoids cause disruption of muscle glucose uptake. Therefore, the suggested opening of skeletal $\mathrm{K}_{\mathrm{ATP}}$ channels in glucocorticoid induced myopathy could cause the deteriorated hyperglycemic state in nicorandil treated group $(35,47)$.

\section{Conclusion}

It seems that blocking of $\mathrm{K}_{\mathrm{ATP}}$ channels by administration of glibenclamide exhibited a protective role against glucocorticoid-induced myopathy in a rat model, while opening them by nicorandil seems to worsen the myopathic condition. Such protective role seems to be due to the enhancement of IGF-1 expression and hence the entire anabolic pathway, the improved intracellular potassium uptake with improved insulin level and glycemic condition. Yet, no improvement of the oxidative state with both drugs might allude to exclusion of the $\mathrm{K}_{\mathrm{ATP}}$ channels from induction of oxidative stress in the case of glucocorticoid induced myopathy. Moreover, these diverse findings in case of opening and blocking the $\mathrm{K}_{\mathrm{ATP}}$ channels imply a possible role for increased $\mathrm{K}_{\mathrm{ATP}}$ channels activity in the induction of glucocorticoid-induced myopathy.

\section{Acknowledgements}

We acknowledge Physiology Department and Medical Experimental Research Center of Mansoura University for their significant assistance in the experimental portion of the research.

\section{References}

1. Minetto MA, D'Angelo V, Arvat E, Kesari S. Diagnostic work-up in steroid myopathy. Endocrine. 2018;60(2):219-23.

2. Pereira RMR, Freire de Carvalho J. Glucocorticoid-induced myopathy. Joint Bone Spine. 2011;78(1):41-4.

\section{Schakman O, Kalista S, Barbé C, Loumaye}

A, Thissen J-P. Glucocorticoid-induced skeletal muscle atrophy. The international journal of biochemistry \& cell biology. 2013;45(10):216372.

\section{Berr CM, Stieg MR, Deutschbein T, Quinkler}

M, Schmidmaier R, Osswald A, et al. Persistence of myopathy in Cushing's syndrome: evaluation of the German Cushing's Registry. European journal of endocrinology. 2017;176(6):737-46.

\section{Schakman O, Gilson H, Thissen JP.} Mechanisms of glucocorticoid-induced myopathy. The Journal of endocrinology. 2008;197(1):1-10.

6. Gupta A, Gupta Y. Glucocorticoid-induced myopathy: Pathophysiology, diagnosis, and treatment. Indian journal of endocrinology and metabolism. 2013;17(5):913.

7. Grzanka A, Misiołek M, Golusiński W, Jarząb J. Molecular mechanisms of glucocorticoids action: implications for treatment of rhinosinusitis and nasal polyposis. European Archives of Oto-Rhino-Laryngology. 2011;268(2):247-53.

8. Tinker A, Aziz Q, Li Y, Specterman M. ATP-Sensitive Potassium Channels and Their Physiological and Pathophysiological Roles. Comprehensive Physiology. 2018;8:1463-511. 
9. Rubaiy HN. The therapeutic agents that target ATP-sensitive potassium channels. Acta pharmaceutica. 2016;66(1):23-34.

10. Jahangir A, Terzic A. KATP channel therapeutics at the bedside. J Mol Cell Cardiol. 2005;39(1):99-112.

11. Flagg TP, Enkvetchakul D, Koster JC, Nichols CG. Muscle KATP Channels: Recent Insights to Energy Sensing and Myoprotection. Physiological Reviews. 2010;90(3):799-829.

12. Tricarico D, Selvaggi M, Passantino G, De Palo P, Dario C, Centoducati $\mathbf{P}$, et al. ATP sensitive potassium channels in the skeletal muscle function: involvement of the KCNJ11 (Kir6. 2) gene in the determination of mechanical Warner Bratzer shear force. Frontiers in Physiology. 2016;7:167.

13. Huang Y, Chen K, Ren Q, Yi L, Zhu J, Zhang Q, et al. Dihydromyricetin attenuates dexamethasone-induced muscle atrophy by improving mitochondrial function via the PGC-1 $\alpha$ pathway. Cellular Physiology and Biochemistry. 2018;49(2):758-79.

14. Parasuraman S, Raveendran R, Kesavan R. Blood sample collection in small laboratory animals. Journal of pharmacology \& pharmacotherapeutics. 2010;1(2):87.

15. Head SI, Arber M. An active learning mammalian skeletal muscle lab demonstrating contractile and kinetic properties of fast-and slowtwitch muscle. Advances in physiology education. 2013;37(4):405-14.

16. Sandor NL. A murine model of glucocorticoid myopathy alleviation using androgen therapy. https://hdl.handle.net/2144/13732:

Boston University; 2015.
17. Huang Y, Chen K, Ren Q, Yi L, Zhu J, Zhang Q, et al. Dihydromyricetin Attenuates Dexamethasone-Induced Muscle Atrophy by Improving Mitochondrial Function via the PGC1alpha Pathway. Cellular physiology and biochemistry : international journal of experimental cellular physiology, biochemistry, and pharmacology. 2018;49(2):758-79.

18. Müller R, Kugelberg E. Myopathy in Cushing's syndrome. Journal of neurology, neurosurgery, and psychiatry. 1959;22(4):314-9.

19. Lee MJ, Lee JS, Lee MC. Apoptosis of skeletal muscle on steroid-induced myopathy in rats. Journal of Korean medical science. 2001;16(4):467.

20. Peters SA, Kley RA. Toxic and Drug-Induced Myopathies: Clinical Scenario and Imaging. Clinical Neuroradiology: The ESNR Textbook. 2019:1-15.

21. Zhu Z, Sierra A, Burnett CM-L, Chen B, Subbotina E, Koganti SRK, et al. Sarcolemmal ATP-sensitive potassium channels modulate skeletal muscle function under low-intensity workloads. The Journal of General Physiology. 2014;143(1):119-34.

\section{Sanchez-Duarte E, Trujillo X, Cortés-Rojo} C, Saavedra-Molina A, Camargo G, Hernández $\mathbf{L}$, et al. Nicorandil improves post-fatigue tension in slow skeletal muscle fibers by modulating glutathione redox state. Journal of bioenergetics and biomembranes. 2017;49(2):159-70.

23. Chowdhry V, Mohanty B. Intractable hyperkalemia due to nicorandil induced potassium channel syndrome. Annals of cardiac anaesthesia. 2015;18(1):101.

24.Chasioti D, Yao X, Zhang P, Lerner S, Quinney SK, Ning X, et al. Mining directional 
drug interaction effects on myopathy using the FAERS database. IEEE journal of biomedical and health informatics. 2018;23(5):2156-63.

25. Mele A, Calzolaro S, Cannone G, Cetrone M, Conte D, Tricarico D. Database search of spontaneous reports and pharmacological investigations on the sulfonylureas and glinides-induced atrophy in skeletal muscle. Pharmacology research \& perspectives. 2014;2(1):e00028.

\section{Inder WJ, Jang C, Obeyesekere VR, Alford}

FP. Dexamethasone administration inhibits skeletal muscle expression of the androgen receptor and IGF-1-implications for steroid-induced myopathy. Clinical endocrinology. 2010;73(1):126-32.

\section{Manoj M, Sahoo RR, Hazarika K, Bafna $P$,}

Wakhlu A. Drug-induced myopathy. Indian Journal of Rheumatology. 2019;14(5):27.

28. Abeer FM. Ferulic Acid Promotes Growth of Both Fast Glycolytic and Slow Oxidative Skeletal Muscles in Corticosteroid-Induced Rat Myopathy. The Medical Journal of Cairo University. 2019;87(June):1703-15.

29. Haba M, Hatakeyama N, Kinoshita H, Teramae H, Azma T, Hatano Y, et al. The Modulation of Vascular ATP-Sensitive $\mathrm{K}+$ Channel Function via the Phosphatidylinositol 3Kinase-Akt Pathway Activated by Phenylephrine. Journal of Pharmacology and Experimental Therapeutics. 2010;334(2):673-8.

30. Stitt TN, Drujan D, Clarke BA, Panaro F, Timofeyva Y, Kline WO, et al. The IGF1/PI3K/Akt pathway prevents expression of muscle atrophy-induced ubiquitin ligases by inhibiting FOXO transcription factors. Molecular cell. 2004;14(3):395-403.

31. Foster MN, Coetzee WA. KATP channels in the cardiovascular system. Physiological reviews. 2016;96(1):177-252.

32. Philip-Couderc P, Tavares NI, Roatti A, Lerch R, Montessuit C, Baertschi AJ. Forkhead transcription factors coordinate expression of myocardial KATP channel subunits and energy metabolism. Circulation research. 2008;102(2):e20-e35.

33. Dumas J-F, Bielicki G, Renou J-P, Roussel D, Ducluzeau P-H, Malthièry $\mathbf{Y}$, et al. Dexamethasone impairs muscle energetics, studied by $31 \mathrm{P}$ NMR, in rats. Diabetologia. 2005;48(2):328-35.

\section{Liu J, Peng Y, Wang X, Fan Y, Qin C, Shi} L, et al. Mitochondrial dysfunction launches dexamethasone-induced skeletal muscle atrophy via AMPK/FOXO3 signaling. Molecular pharmaceutics. 2016;13(1):73-84.

35. Ferrau F, Korbonits M. Metabolic comorbidities in Cushing's syndrome. European Journal of Endocrinology. 2015;173(4):M133M57.

36. Sierra A, Subbotina E, Zhu Z, Gao Z, Koganti SRK, Coetzee WA, et al. Disruption of ATP-sensitive potassium channel function in skeletal muscles promotes production and secretion of musclin. Biochemical and biophysical research communications. 2016;471(1):129-34.

37. Konno S. Hydroxyl radical formation in skeletal muscle of rats with glucocorticoid-induced myopathy. Neurochemical research. 2005;30(5):669-75. 
38. Baird MF, Graham SM, Baker JS, Bickerstaff GF. Creatine-kinase-and exerciserelated muscle damage implications for muscle performance and recovery. Journal of nutrition and metabolism. 2012;2012.

39. Duty S, Allen D. The effects of glibenclamide on tetanic force and intracellular calcium in normal and fatigued mouse skeletal muscle. Experimental Physiology: Translation and Integration. 1995;80(4):529-41.

40. Burton F, Smith G. The effect of cromakalim on intracellular $[\mathrm{Ca} 2+]$ in isolated rat skeletal muscle during fatigue and metabolic blockade. Experimental Physiology: Translation and Integration. 1997;82(3):469-83.

41. Renaud J. Modulation of force development by $\mathrm{Na}+, \mathrm{K}+, \mathrm{Na}+\mathrm{K}+$ pump and KATP channel during muscular activity. Canadian journal of applied physiology. 2002;27(3):296-315.

42. Cifelli C, Boudreault L, Gong B, Bercier JP, Renaud JM. Contractile dysfunctions in ATP-dependent $\mathrm{K}+$ channel-deficient mouse muscle during fatigue involve excessive depolarization and $\mathrm{Ca} 2+$ influx through L-type $\mathrm{Ca} 2+$ channels. Experimental physiology. 2008;93(10):1126-38.

43. Zhu Z, Sierra A, Burnett CM-L, Chen B, Subbotina E, Koganti SRK, et al. Sarcolemmal ATP-sensitive potassium channels modulate skeletal muscle function under low-intensity workloads. Journal of General Physiology. 2014;143(1):119-34.

\section{Gong B, Legault D, Miki T, Seino S, Renaud}

J-M. KATP channels depress force by reducing action potential amplitude in mouse EDL and soleus muscle. American Journal of PhysiologyCell Physiology. 2003;285(6):C1464-C74.

45. Sejersted OM, Sjøgaard G. Dynamics and consequences of potassium shifts in skeletal muscle and heart during exercise. Physiological Reviews. 2000;80(4):1411-81.

46. Weiner ID, Wingo CS. Hypokalemia-consequences, causes, and correction. Journal of the American Society of Nephrology. 1997;8(7):1179-88.

\section{Miki T, Minami K, Zhang L, Morita M,} Gonoi T, Shiuchi $\mathbf{T}$, et al. ATP-sensitive potassium channels participate in glucose uptake in skeletal muscle and adipose tissue. American Journal of Physiology-Endocrinology and Metabolism. 2002;283(6):E1178-E84. 